\title{
Synthesis and antimicrobial activity of some oxazaphosphinine oxides
}

\author{
B. Siva Kumar, A. U. Ravi Sankar, G. Chandra Sekhar Reddy, M. V. Narayana Reddy, C. \\ Devendranath Reddy, and C. Suresh Reddy*
}

Department of Chemistry Sri Venkateswara University, Tirupati, 517502, India

E-mail: csureshsvu@yahoo.com

\begin{abstract}
A new family of phosphorus heterocycle, namely 2-substituted 3-[4-(2-oxo-3,4-dihydro- $2 \lambda^{5}$ benzo[e][1,3,2]oxazaphosphinin-3-yl)phenyl]-3,4-dihydrobenzo[e][1,3,2]oxazaphosphinin-2oxides $(\mathbf{5 a}-\mathbf{j})$ has been synthesized by the condensation of 2-[[4-(2-hydroxy-benzylamino)phenylamino]methyl]phenol (1) with phosphorus oxychloride in presence of triethylamine in dry tetrahydrofuran, followed by the reaction with various phenols $\mathbf{3 a}-\mathbf{j}$. Alternatively, some of these compounds 5a-e were prepared by the cyclocondensation of $\mathbf{1}$ with aryl phosphorodichloridates 4a-e. All title compounds were characterized by elemental and spectral analyses. Their antimicrobial activity was also evaluated.
\end{abstract}

Keywords: Oxazaphosphinine, arylphosphorodichloridates, antimicrobial analysis, spectral analysis

\section{Introduction}

Organophosphorus heterocycles containing $\mathrm{O}$ and $\mathrm{N}$ in a six-membered ring have gained much attention ever since cyclophosphamide was discovered as anti-cancer drug. ${ }^{1}$ Compounds of this class also have high anti-tumor activity, ${ }^{2-5}$ significant bioactivity, ${ }^{6}$ and outstanding medicinal

properties. $^{7}$ The significant activity of all these compounds was accredited to the presence of sixmembered heterocyclic rings. In our present research, synthesis of compounds containing two such rings was accomplished successfully. All compounds were characterized by elemental, IR, ${ }^{1} \mathrm{H},{ }^{13} \mathrm{C}$ and ${ }^{31} \mathrm{P}$ NMR and mass spectral analysis. Their antimicrobial activity was also evaluated. 


\section{Results and Discussion}

Cyclocondensation of 2-[[4-(2-hydroxybenzylamino)phenylamino]methyl]-phenol (1) with phosphorus oxychloride in presence of triethylamine in dry tetrahydrofuran at $40-50{ }^{\circ} \mathrm{C}$ afforded 2-chloro-3[4-(2-chloro-2-oxo-3,4-dihydro-2 $\lambda^{5}$-benzo[e][1,3,2] oxazaphosphinine-2-oxide which upon subsequent reaction with various phenols $\mathbf{3 a}-\mathbf{j}$ gave 3-[4-(2-aryloxy-2-oxo-3,4dihydrobenzo[e][1,3,2] oxazaphosphinine-3-yl)phenyl]-2-aryloxy-3,4-dihydrobenzo-

$[e][1,3,2]$ oxazaphosphinine-2-oxides $(\mathbf{5 a}-\mathbf{j})$ in good yields. Some products 5 were prepared by condensation of $\mathbf{1}$ with aryl phosphorodichloridates ${ }^{8} \mathbf{4 a}-\mathbf{e}$ in the presence of triethylamine in dry THF. The yields of the products obtained by both routes are comparable. Direct condensation of compound 1 with aryl phosphorodichoridates (4a-e) afforded good yields more conveniently than the former method because compound $\mathbf{2}$ is highly moisture sensitive and difficult to handle. All compounds were purified by recrystallizing from 2-propanol and were characterized by elemental, IR, ${ }^{1} \mathrm{H},{ }^{13} \mathrm{C},{ }^{31} \mathrm{P} \mathrm{NMR}$, and partly by mass spectral analyses.

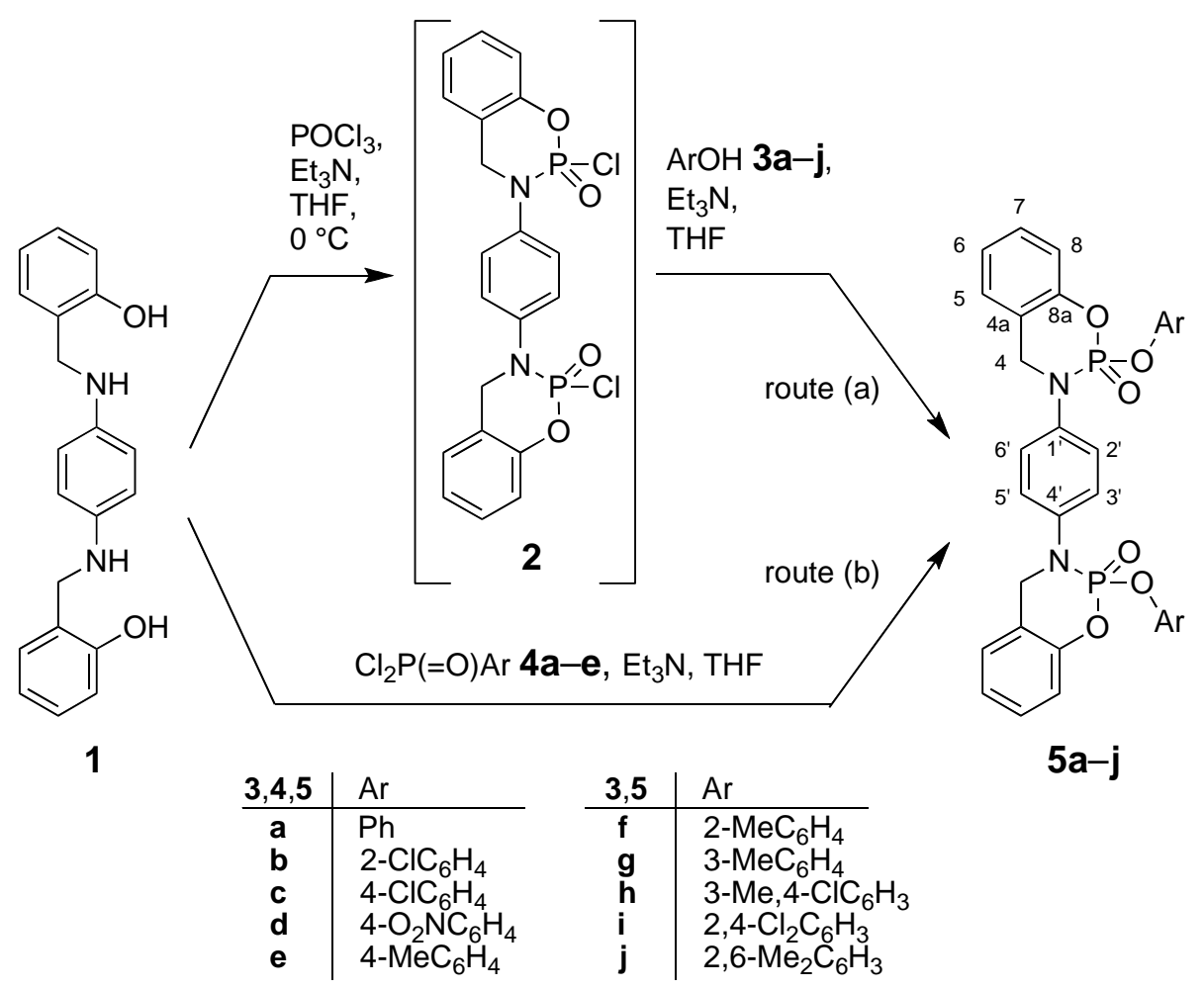

\section{Scheme 1}

All compounds 5a-j exhibit characteristic IR absorption bands in the regions 1196-1231, 1109-1131, and 908-921 $\mathrm{cm}^{-1}$ indicative of $(\mathrm{P}=\mathrm{O}),{ }^{9-11}(\mathrm{C}-\mathrm{O})$, and $(\mathrm{P}-\mathrm{O}),{ }^{12,13}$ respectively. ${ }^{1} \mathrm{H}$ 
NMR spectra of compounds 5a-j exhibit multiplets in the range $\delta$ 6.19-8.17 for aromatic protons. Methylene protons resonate as multiplets at $\delta$ 4.41-5.09 indicating their nonequivalence ${ }^{14}$ and coupling with phosphorus in the six-membered chair-like conformation of the benzoxazaphosphinine system.

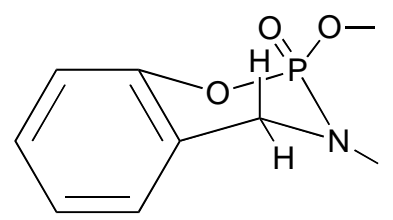

In the ${ }^{13} \mathrm{C}$ NMR spectra of compounds $\mathbf{5 a}-\mathbf{j}$, the oxygen-bearing C-8a exhibits signals in the range of $\delta 149.5-153.9$. The signal in the region $\delta 129.2-129.7$ is assigned to C-4a. Signals in the ranges of $\delta$ 128.4-129.0 and 123.3-124.4 are assigned to C-5 and C-6, respectively. The two signals exhibited at $\delta$ 132.3-133.0 and 115.8-118.9 are attributed to C-1',4' and C-2',3',5',6', respectively. Methylene C-4 appears as a doublet in the range $\delta 45.3-46.3\left({ }^{2} J_{\mathrm{PC}}=124.78-132.43\right.$ $\mathrm{Hz}){ }^{31} \mathrm{P}$ NMR chemical shift values ${ }^{15}$ of these compounds appears within the range of $\delta 1.23-$ 6.84. The above data suggest that the two benzoxazaphosphonine rings are present in the same chemical and magnetic environment.

\section{Antimicrobial activity}

Compounds 5a-j were screened for their antibacterial activity ${ }^{16,17}$ against gram-positive Staphylococcus aureus and gram-negative Klebsiella pneumoniae by the disc-fusion method in nutrient agar medium at two concentrations (200, $400 \mathrm{ppm})$ in DMF. These solutions were added to each filter disc, and the plates were incubated at $35^{\circ} \mathrm{C}$ and examined for zone of inhibition around each disc after $24 \mathrm{~h}$. Results were compared with the activity of the standard antibiotic Penicillin. Antifungal activities were evaluated against Pellicularia solmanicolor and Macrophomina phaseolina at two concentrations ${ }^{18}(200,400 \mathrm{ppm})$ using Griseofulvin as reference compound. Fungal cultures were grown on potato dextrose broth at $25{ }^{\circ} \mathrm{C}$ and spore suspension was adjusted to $10^{5}$ spore $/ \mathrm{mL}$. All compounds exhibited antimicrobial activity comparable with that of reference compounds. Some of the compounds showed high acivity againist both the bacteria and fungi. 
Table 1. Antibacterial and antifungal activities of $\mathbf{5 a}-\mathbf{j}$

\begin{tabular}{|c|c|c|c|c|c|c|c|c|}
\hline \multirow{3}{*}{ Compound } & \multicolumn{8}{|c|}{ Zone of inhibition } \\
\hline & \multicolumn{2}{|c|}{$\begin{array}{c}\text { Staphylococcus } \\
\text { aureus }\end{array}$} & \multicolumn{2}{|c|}{$\begin{array}{c}\text { Klebsiella } \\
\text { pneumoniae }\end{array}$} & \multicolumn{2}{|c|}{$\begin{array}{l}\text { Pellicularia } \\
\text { solamnicolor }\end{array}$} & \multicolumn{2}{|c|}{$\begin{array}{c}\text { Macrophomina } \\
\text { phaseolina }\end{array}$} \\
\hline & $200^{\mathrm{a}}$ & $400^{\mathrm{a}}$ & $200^{\mathrm{a}}$ & $400^{\mathrm{a}}$ & $200^{\mathrm{a}}$ & $400^{\mathrm{a}}$ & $200^{\mathrm{a}}$ & $400^{\mathrm{a}}$ \\
\hline $5 \mathbf{a}$ & 23 & 46 & 21 & 39 & 21 & 47 & 20 & 46 \\
\hline $5 b$ & 21 & 43 & 24 & 45 & 20 & 44 & 18 & 42 \\
\hline $5 c$ & 19 & 37 & 20 & 41 & 22 & 50 & 24 & 50 \\
\hline $5 d$ & 17 & 36 & 22 & 43 & 18 & 42 & 16 & 39 \\
\hline $5 e$ & 20 & 42 & 20 & 40 & 24 & 51 & 25 & 48 \\
\hline $5 f$ & 15 & 31 & 17 & 36 & 19 & 44 & 20 & 45 \\
\hline $5 g$ & 21 & 42 & 16 & 34 & 21 & 46 & 17 & 40 \\
\hline $5 \mathrm{~h}$ & 25 & 48 & 22 & 43 & 22 & 49 & 23 & 50 \\
\hline $5 \mathbf{i}$ & 19 & 38 & 16 & 36 & 20 & 48 & 19 & 45 \\
\hline $5 j$ & 21 & 41 & 19 & 40 & 19 & 43 & 17 & 41 \\
\hline Penicillin ${ }^{\mathrm{b}}$ & 22 & 41 & 24 & 46 & & & & \\
\hline Griseofulvin $^{\mathrm{b}}$ & & & & & 23 & 44 & 24 & 47 \\
\hline
\end{tabular}

${ }^{\mathrm{a}}$ In DMF, concentration in ppm. ${ }^{\mathrm{b}}$ Standard reference.

\section{Experimental Section}

General Procedures. Melting points were determined in open capillary tubes on a Mel-Temp apparatus. IR spectra were recorded on a Perkin Elmer 1000 unit. The ${ }^{1} \mathrm{H},{ }^{13} \mathrm{C}$ and ${ }^{31} \mathrm{P}$ NMR spectra were recorded on Varian Gemini 300 and Varian AMX 400 NMR spectrometers operating at 300 or $400 \mathrm{MHz}\left({ }^{1} \mathrm{H}\right), 75.46$ or $100.57 \mathrm{MHz}\left({ }^{13} \mathrm{C}\right)$ and $121.7 \mathrm{MHz}\left({ }^{31} \mathrm{P}\right)$. Mass spectra were recorded by Fast Atom Bombardment mass spectrometer. All compounds were dissolved in DMSO- $d_{6}$, chemical shifts are referenced to TMS $\left({ }^{1} \mathrm{H},{ }^{13} \mathrm{C}\right)$ and $85 \% \mathrm{H}_{3} \mathrm{PO}_{4}\left({ }^{31} \mathrm{P}\right)$. Microanalytical data were obtained from Central Drug Research Institute, Lucknow, India.

\section{3-[4-(2-Phenoxy-2-oxo-3,4-dihydro-2 $\lambda^{5}$-benzo[e][1,3,2]oxazaphosphinin-3-yl)-phenyl]-2- phenoxy-3,4-dihydrobenzo[e][1,3,2] oxazaphosphinin-2-oxide (5a). Typical procedure}

(a) To a stirred solution of 2-[[4-(2-hydroxybenzylamino)phenylamino]methyl]phenol (1, 1.65 g, $5 \mathrm{mmol})$ in dry THF $(25 \mathrm{~mL})$ was added dropwise phosphorus oxychloride $(0.932 \mathrm{~g}, 10 \mathrm{mmol})$ in dry THF $(10 \mathrm{~mL})$ in the presence of triethylamine $(2.02 \mathrm{~g}, 20 \mathrm{mmol})$ at $0{ }^{\circ} \mathrm{C}$. After addition, the temperature was slowly raised to $50-60{ }^{\circ} \mathrm{C}$; the progress of the reaction was monitored by TLC. Triethylamine hydrochloride was sucked off. To the filtrate was added freshly distilled phenol (3a, $0.47 \mathrm{~g}, 5 \mathrm{mmol})$ in dry THF $(15 \mathrm{~mL})$ and triethylamine $(1.01 \mathrm{~g}, 10 \mathrm{mmol})$, and the progress of the reaction was monitored by TLC. Triethylamine hydrochloride was filtered off, the solvent 
was removed under reduced pressure. The crude product was recrystallized from 2-propanol to get colorless crystals 5a (1.93 g, 65\%).

(b) To a stirred solution of 1 (1.65 g, $5 \mathrm{mmol})$ in dry THF $(25 \mathrm{~mL})$ was added dropwise phenyl phosphorodichloridate $(4 \mathbf{a}, 2.1 \mathrm{~g}, 10 \mathrm{mmol})$ in dry THF $(15 \mathrm{~mL})$ in the presence of triethylamine $(2.02 \mathrm{~g}, 20 \mathrm{mmol})$ at $0{ }^{\circ} \mathrm{C}$. After addition, the temperature was maintained between $50-55^{\circ} \mathrm{C}$, and the progress of the reaction was monitored by TLC. The crude product was recrystallized from 2-propanol to yield 5a $(2.02 \mathrm{~g}, 68 \%)$.

5a. Mp $120-122{ }^{\circ} \mathrm{C}$. IR (KBr): $\tilde{v} 1226(\mathrm{P}=\mathrm{O}), 1128(\mathrm{O}-\mathrm{C}), 916(\mathrm{P}-\mathrm{O}) \mathrm{cm}^{-1}$. ${ }^{1} \mathrm{H}$ NMR $(300 \mathrm{MHz}$, DMSO-d $\left.d_{6}\right): \delta 6.70-7.81(\mathrm{~m}, 22 \mathrm{H}), 4.50-4.70(\mathrm{~m}, 4 \mathrm{H}) .{ }^{13} \mathrm{C}$ NMR $\left(100 \mathrm{MHz}, \mathrm{DMSO}-d_{6}\right): \delta 45.4$ $\left(\mathrm{d}, J_{\mathrm{PC}}=129.30 \mathrm{~Hz}, \mathrm{C}-4\right), 129.2$ (C-4a), 128.7 (C-5), 124.3 (C-5), 127.9 (C-7), 123.9 (C-8), $149.5(\mathrm{C}-8 \mathrm{a}), 132.6$ (C-1', C-4'), $118.6\left(\mathrm{C}-2^{\prime}, \mathrm{C}-3^{\prime}, \mathrm{C}-5^{\prime}, \mathrm{C}-6^{\prime}\right), 157.4\left(1-\mathrm{C}_{\mathrm{Ar}}\right), 114.9\left(2-\mathrm{C}_{\mathrm{Ar}}\right)$, $128.1\left(3-\mathrm{C}_{\mathrm{Ar}}\right), 130.4\left(4-\mathrm{C}_{\mathrm{Ar}}\right), 128.1\left(5-\mathrm{C}_{\mathrm{Ar}}\right), 114.9\left(6-\mathrm{C}_{\mathrm{Ar}}\right) .{ }^{31} \mathrm{P}$ NMR $\left(161.98 \mathrm{MHz}, \mathrm{DMSO}-d_{6}\right): \delta$ 2.98. FAB-MS: m/z (\%) $596\left(8, \mathrm{M}^{+\bullet}\right), 508$ (27), 504 (19), 456 (21), 368 (100), 276 (9), 248 (35). Anal. calcd. for $\mathrm{C}_{32} \mathrm{H}_{26} \mathrm{~N}_{2} \mathrm{O}_{6} \mathrm{P}_{2}$ : C, 64.43; H, 4.39; N, 4.69. Found: C, 64.52; H, 4.46; N, 4.47.

$\mathbf{5 b}$-e were prepared by the above two procedures, $\mathbf{5 f}-\mathbf{j}$ were prepared following route (a).

3-[4-[2-(2-Chlorophenoxy)-2-oxo-3,4-dihydro-2 $\lambda^{5}$-benzo[e][1,3,2] oxazaphosphinin-3-yl]phenyl]-2-(2-chlorophenoxy)-3,4-dihydrobenzo[e][1,3,2]oxazaphosphine-2-oxide (5b). Route (b): Colorless crystals $(2.12 \mathrm{~g}, 62 \%) ; \mathrm{mp} 159-161{ }^{\circ} \mathrm{C}$. IR (KBr): $\tilde{v} 1212(\mathrm{P}=\mathrm{O}), 1118(\mathrm{O}-\mathrm{C}), 913$ $(\mathrm{P}-\mathrm{O}) \mathrm{cm}^{-1} .{ }^{1} \mathrm{H}$ NMR $\left(300 \mathrm{MHz}, \mathrm{DMSO}-d_{6}\right): \delta 6.31-7.62(\mathrm{~m}, 20 \mathrm{H}), 4.61-4.92(\mathrm{~m}, 4 \mathrm{H}) .{ }^{13} \mathrm{C}$ NMR (100 MHz, DMSO-d $\left.d_{6}\right): \delta 46.3\left(\mathrm{~d}, J_{\mathrm{PC}}=131.12 \mathrm{~Hz}, \mathrm{C}-4\right), 129.5$ (C-4a), 128.7 (C-5), 123.3 (C-6), 127.9 (C-7), 119.3 (C-8), 151.4 (C-8a), 132.3 (C-1', C-4'), 116.3 (C-2', C-3', C-5', C-6'), $155.3\left(1-\mathrm{C}_{\mathrm{Ar}}\right), 123.3\left(2-\mathrm{C}_{\mathrm{Ar}}\right), 128.9\left(3-\mathrm{C}_{\mathrm{Ar}}\right), 131.5\left(4-\mathrm{C}_{\mathrm{Ar}}\right), 126.6\left(5-\mathrm{C}_{\mathrm{Ar}}\right), 130.6\left(6-\mathrm{C}_{\mathrm{Ar}}\right) .{ }^{31} \mathrm{P}$ NMR (161.98 MHz, DMSO- $\left.d_{6}\right): \delta$ 1.23. Anal. calcd. for $\mathrm{C}_{32} \mathrm{H}_{24} \mathrm{Cl}_{2} \mathrm{~N}_{2} \mathrm{O}_{6} \mathrm{P}_{2}: \mathrm{C}, 57.76 ; \mathrm{H}, 3.64 ; \mathrm{N}$, 4.21. Found: C, 57.87; H, 3.69; N, 4.29.

3-[4-[2-(4-Chlorophenoxy)-2-oxo-3,4-dihydro-2 $\lambda^{5}$-benzo[e][1,3,2] oxazaphosphinin-3-yl]phenyl]-2-(4-chlorophenoxy)-3,4-dihydrobenzo[e][1,3,2]oxazaphosphine-2-oxide (5c). Route (b): Colorless crystals (1.93 g, 59\%); mp 154-156 ${ }^{\circ} \mathrm{C}$. IR (KBr): $\tilde{v} 1208(\mathrm{P}=\mathrm{O}), 1131$ (O-C), 917 $(\mathrm{P}-\mathrm{O}) \mathrm{cm}^{-1} .{ }^{1} \mathrm{H}$ NMR $\left(300 \mathrm{MHz}, \mathrm{DMSO}-d_{6}\right): \delta 6.61-7.62(\mathrm{~m}, 20 \mathrm{H}), 4.42-4.62(\mathrm{~m}, 4 \mathrm{H}) .{ }^{13} \mathrm{C}$ NMR (100 MHz, DMSO- $\left.d_{6}\right): \delta 45.9\left(\mathrm{~d}, J_{\mathrm{PC}}=130.19 \mathrm{~Hz}, \mathrm{C}-4\right), 129.6(\mathrm{C}-4 \mathrm{a}), 128.6\left(\mathrm{C}-5{ }^{\prime}\right), 124.1$ (C-6), 127.9 (C-7'), 121.3 (C-8'), 152.4 (C-8a), 132.5 (C-1', C-4'), 116.6 (C-2', C-3', C-5', C-6'), $154.6\left(1-\mathrm{C}_{\mathrm{Ar}}\right), 130.9\left(2-\mathrm{C}_{\mathrm{Ar}}\right), 128.9\left(3-\mathrm{C}_{\mathrm{Ar}}\right), 135.6\left(4-\mathrm{C}_{\mathrm{Ar}}\right), 128.9\left(5-\mathrm{C}_{\mathrm{Ar}}\right), 130.9\left(6-\mathrm{C}_{\mathrm{Ar}}\right) .{ }^{31} \mathrm{P}$ NMR (161.98 MHz, DMSO-d $)$ ): $\delta$ 3.72. Anal. calcd. for $\mathrm{C}_{32} \mathrm{H}_{24} \mathrm{Cl}_{2} \mathrm{~N}_{2} \mathrm{O}_{6} \mathrm{P}_{2}: \mathrm{C}, 57.76 ; \mathrm{H}, 3.64 ; \mathrm{N}$, 4.21. Found: C, 57.84; H, 3.69; N, 4.30.

3-[4-[2-(4-Nitrophenoxy)-2-oxo-3,4-dihydro-2 $\lambda^{5}$-benzo[e][1,3,2]oxazaphosphinin-3-yl]phenyl]-2-(4-nitrophenoxy)-3,4-dihydrobenzo[e][1,3,2]oxazaphosphine-2-oxide (5d). Route (b): Pale yellow crystals $(2.16 \mathrm{~g}, 61 \%)$; mp 139-142 ${ }^{\circ} \mathrm{C}$. IR (KBr): $\tilde{v} 1196(\mathrm{P}=\mathrm{O}), 1114$ (O-C), $911(\mathrm{P}-\mathrm{O}) \mathrm{cm}^{-1} .{ }^{1} \mathrm{H}$ NMR $\left(300 \mathrm{MHz}, \mathrm{DMSO}-d_{6}\right): \delta 6.59-7.81(\mathrm{~m}, 20 \mathrm{H}), 4.84-5.17(\mathrm{~m}, 4 \mathrm{H}) .{ }^{13} \mathrm{C}$ NMR (100 MHz, DMSO- $\left.d_{6}\right): \delta 45.3\left(\mathrm{~d}, J_{\mathrm{PC}}=133.11 \mathrm{~Hz}, \mathrm{C}-4\right), 129.4(\mathrm{C}-4 \mathrm{a}), 128.4(\mathrm{C}-5), 124.2$ (C-6), 128.1 (C-7), 122.9 (C-8), 152.1 (C-8a), 132.7 (C-1', C-4'), 115.4 (C-2', C-3', C-5', C-6'), $156.3\left(1-\mathrm{C}_{\mathrm{Ar}}\right), 117.3\left(2-\mathrm{C}_{\mathrm{Ar}}\right), 127.4\left(3-\mathrm{C}_{\mathrm{Ar}}\right), 141.3\left(4-\mathrm{C}_{\mathrm{Ar}}\right), 127.4\left(5-\mathrm{C}_{\mathrm{Ar}}\right), 117.3\left(6-\mathrm{C}_{\mathrm{Ar}}\right) .{ }^{31} \mathrm{P}$ 
NMR (161.98 MHz, DMSO-d $\left.)_{6}\right): \delta$ 6.84. Anal. calcd. for $\mathrm{C}_{32} \mathrm{H}_{24} \mathrm{~N}_{4} \mathrm{O}_{10} \mathrm{P}_{2}: \mathrm{C}, 55.99 ; \mathrm{H}, 3.52 ; \mathrm{N}$, 8.16. Found: C, 56.12; H, 3.68; N, 8.27.

3-[4-[2-(4-Methylphenoxy)-2-oxo-3,4-dihydro-2 $\lambda^{5}$-benzo[e][1,3,2] oxazaphos-phinin-3yl]phenyl]-2-(4-methylphenoxy)-3,4-dihydrobenzo[e][1,3,2]oxazaphosphine-2-oxide (5e). Route (b): Colorless crystals (1.93 g, 60\%); mp 161-163 ${ }^{\circ} \mathrm{C}$. IR (KBr): $\tilde{v} 1212(\mathrm{P}=\mathrm{O}), 1122(\mathrm{O}-$ C), $918(\mathrm{P}-\mathrm{O}) \mathrm{cm}^{-1} .{ }^{1} \mathrm{H}$ NMR (300 MHz, DMSO-d $): \delta 6.14-7.77(\mathrm{~m}, 26 \mathrm{H}), 4.75-4.94(\mathrm{~m}, 4 \mathrm{H})$, $1.92\left(\mathrm{~m}, 4-\mathrm{H}_{\mathrm{Ar}}, 6 \mathrm{H}\right) .{ }^{13} \mathrm{C}$ NMR $\left(100 \mathrm{MHz}, \mathrm{DMSO}-\mathrm{d}_{6}\right): \delta 46.1\left(\mathrm{~d}, J_{\mathrm{PC}}=132.43 \mathrm{~Hz}, \mathrm{C}-4\right), 129.7$ (C-4a), 129.0 (C-5 ), 124.0 (C-6), 128.6 (C-7), 122.2 (C-8), 153.4 (C-8a), 133.0 (C-1', C-4'), 117.1 (C-2', C-3', C-5', C-6'), $156.3\left(1-\mathrm{C}_{\mathrm{Ar}}\right), 119.3\left(2-\mathrm{C}_{\mathrm{Ar}}\right), 128.9\left(3-\mathrm{C}_{\mathrm{Ar}}\right), 139.4\left(4-\mathrm{C}_{\mathrm{Ar}}\right), 128.9$ $\left(5-\mathrm{C}_{\mathrm{Ar}}\right), 130.0\left(6-\mathrm{C}_{\mathrm{Ar}}\right), 20.9\left(2 \mathrm{C}, 2 \mathrm{CH}_{3}\right) .{ }^{31} \mathrm{P}$ NMR $\left(161.98 \mathrm{MHz}, \mathrm{DMSO}-d_{6}\right): \delta 2.61$. FAB-MS: m/z (\%) 624 (12, M $\left.{ }^{+\bullet}\right), 536$ (19), 518 (21), 470 (33), 382 (100), 322 (31), 260 (13), 140 (36). Anal. calcd. for $\mathrm{C}_{34} \mathrm{H}_{30} \mathrm{~N}_{2} \mathrm{O}_{12} \mathrm{P}_{2}$ : C, 65.39; H, 4.84; N, 4.49. Found: C, 65.47; H, 4.92; N, 4.57.

3-[4-[2-(2-Methylphenoxy)-2-oxo-3,4-dihydro-2 $\lambda^{5}$-benzo[e][1,3,2] oxazaphos-phinin-3yl]phenyl]-2-(2-methylphenoxy)-3,4-dihydrobenzo[e][1,3,2]oxazaphosphine-2-oxide (5f). Route (a): Pale brown crystals (2.15 g, 67\%); mp 144-146 ${ }^{\circ} \mathrm{C}$. IR (KBr): $\tilde{v} 1231(\mathrm{P}=\mathrm{O}), 1109$ (O-C), $912(\mathrm{P}-\mathrm{O}) \mathrm{cm}^{-1} .{ }^{1} \mathrm{H}$ NMR (300 MHz, DMSO-d $): \delta 6.54-8.13(\mathrm{~m}, 26 \mathrm{H}), 4.67-4.81(\mathrm{~m}$, $4 \mathrm{H}), 2.10-2.19\left(\mathrm{~m}, 2-\mathrm{H}_{\mathrm{Ar}}, 6 \mathrm{H}\right) .{ }^{13} \mathrm{C}$ NMR $\left(100 \mathrm{MHz}, \mathrm{DMSO}-d_{6}\right): \delta 45.8\left(\mathrm{~d}, J_{\mathrm{PC}}=124.78 \mathrm{~Hz}, \mathrm{C}-\right.$ 4), 129.5 (C-4a), 128.7 (C-5), 123.9 (C-6), 128.1 (C-7), 122.4 (C-8), 153.9 (C-8a), 132.9 (C-1', C-4'), 116.2 (C-2', C-3', C-5', C-6'), $158.3\left(1-\mathrm{C}_{\mathrm{Ar}}\right), 118.5\left(2-\mathrm{C}_{\mathrm{Ar}}\right), 129.6\left(3-\mathrm{C}_{\mathrm{Ar}}\right), 131.9\left(4-\mathrm{C}_{\mathrm{Ar}}\right)$, $124.9\left(5-\mathrm{C}_{\mathrm{Ar}}\right), 129.4\left(6-\mathrm{C}_{\mathrm{Ar}}\right), 15.9\left(2 \mathrm{C}, 2 \mathrm{CH}_{3}\right) .{ }^{31} \mathrm{P}$ NMR $\left(161.98 \mathrm{MHz}, \mathrm{DMSO}-d_{6}\right): \delta 3.49$. Anal. calcd. for $\mathrm{C}_{34} \mathrm{H}_{30} \mathrm{~N}_{2} \mathrm{O}_{6} \mathrm{P}_{2}$ : C, 65.39; H, 4.84; N, 4.49. Found: C, 65.47; H, 4.92; N, 4.57. 3-[4-[2-(3-Methylphenoxy)-2-oxo-3,4-dihydro-2 $\lambda^{5}$-benzo[e][1,3,2]oxazaphos-phinin-3yl]phenyl]-2-(2-methylphenoxy)-3,4-dihydrobenzo[e][1,3,2]oxazaphosphine-2-oxide Route (a): Pale brown crystals (2.05 g, 64\%); mp 150-152 ${ }^{\circ} \mathrm{C}$. IR (KBr): $\tilde{v} 1204(\mathrm{P}=\mathrm{O}), 1121$ (O-C), $921(\mathrm{P}-\mathrm{O}) \mathrm{cm}^{-1}$. ${ }^{1} \mathrm{H}$ NMR (300 MHz, DMSO-d $): \delta 6.39-7.97$ (m, 26H), 4.72-4.99 (m, $4 \mathrm{H}), 1.99\left(\mathrm{~m}, 3-\mathrm{H}_{\mathrm{Ar}}, 6 \mathrm{H}\right) .{ }^{13} \mathrm{C} \mathrm{NMR}\left(100 \mathrm{MHz}, \mathrm{DMSO}-d_{6}\right): \delta 45.9\left(\mathrm{~d}, J_{\mathrm{PC}}=127.55 \mathrm{~Hz}, \mathrm{C}-4\right)$, 129.6 (C-4a), 128.6 (C-5 ), 124.4 (C-6), 128.1 (C-7), 123.1 (C-8), 151.4 (C-8a), 132.4 (C-1', C$\left.4^{\prime}\right), 116.7$ (C-2', C-3', C-5', C-6'), $155.3\left(1-\mathrm{C}_{\mathrm{Ar}}\right), 116.3\left(2-\mathrm{C}_{\mathrm{Ar}}\right), 136.5\left(3-\mathrm{C}_{\mathrm{Ar}}\right), 132.7\left(4-\mathrm{C}_{\mathrm{Ar}}\right)$, $128.9\left(5-\mathrm{C}_{\mathrm{Ar}}\right), 126.6\left(6-\mathrm{C}_{\mathrm{Ar}}\right), 20.1\left(2 \mathrm{C}, 2 \mathrm{CH}_{3}\right) .{ }^{31} \mathrm{P}$ NMR (161.98 MHz, DMSO-d $\left.d_{6}\right): \delta 3.62$. Anal. calcd. for $\mathrm{C}_{34} \mathrm{H}_{30} \mathrm{~N}_{2} \mathrm{O}_{6} \mathrm{P}_{2}$ : C, 65.39; H, 4.84; N, 4.49. Found: C, 65.51; H, 4.98; N, 4.61.

3-[4-[2-(4-Chloro-3-methylphenoxy)-2-oxo-3,4-dihydro-2 $\lambda^{5}$-benzo[e][1,3,2]oxazaphosphinin-3-yl]phenyl]-2-(4-chloro-3-methylphenoxy)-3,4-dihydrobenzo[e][1,3,2]oxazaphosphine-2-oxide (5h). Route (a): Pale brown crystals $(2.22 \mathrm{~g}, 62 \%)$; mp 126-128 ${ }^{\circ} \mathrm{C}$. IR $(\mathrm{KBr}): \tilde{v} 1210(\mathrm{P}=\mathrm{O}), 1119(\mathrm{O}-\mathrm{C}), 908(\mathrm{P}-\mathrm{O}) \mathrm{cm}^{-1} .{ }^{1} \mathrm{H}$ NMR $\left(300 \mathrm{MHz}, \mathrm{DMSO}-d_{6}\right): \delta$ 6.51$7.64(\mathrm{~m}, 24 \mathrm{H}), 4.50-4.71(\mathrm{~m}, 4 \mathrm{H}), 1.93(\mathrm{~m}, 6 \mathrm{H}) .{ }^{13} \mathrm{C}$ NMR $\left(100 \mathrm{MHz}, \mathrm{DMSO}-d_{6}\right): \delta 45.9\left(\mathrm{~d}, J_{\mathrm{PC}}\right.$ $=128.41 \mathrm{~Hz}, \mathrm{C}-4$ ), 129.3 (C-4a), 128.4 (C-5), 123.9 (C-6), 128.1 (C-7), 122.4 (C-8), 152.7 (C8a), 132.6 (C-1', C-4'), 116.7 (C-2', C-3', C-5', C-6'), $154.3\left(1-\mathrm{C}_{\mathrm{Ar}}\right), 117.9\left(2-\mathrm{C}_{\mathrm{Ar}}\right), 128.9\left(3-\mathrm{C}_{\mathrm{Ar}}\right)$, $\left(4-\mathrm{C}_{\mathrm{Ar}}\right), 126.8\left(5-\mathrm{C}_{\mathrm{Ar}}\right), 128.1\left(6-\mathrm{C}_{\mathrm{Ar}}\right), 13.4\left(2 \mathrm{C}, 2 \mathrm{CH}_{3}\right) .{ }^{31} \mathrm{P}$ NMR $\left(161.98 \mathrm{MHz}, \mathrm{DMSO}-d_{6}\right): \delta$ 4.62. Anal. calcd. for $\mathrm{C}_{34} \mathrm{H}_{28} \mathrm{Cl}_{2} \mathrm{~N}_{2} \mathrm{O}_{6} \mathrm{P}_{2}$ : C, 58.89; H, 4.07; N, 4.04. Found: $\mathrm{C}, 58.97 ; \mathrm{H}, 4.15 ; \mathrm{N}$, 4.13 . 


\section{3-[4-[2-(2,4-Dichlorophenoxy)-2-oxo-3,4-dihydro-2 $\lambda^{5}$-benzo[e][1,3,2]oxazaphosphinin-3-} yl]phenyl]-2-(2,4-dichlorophenoxy)-3,4-dihydrobenzo[e][1,3,2]oxazaphosphine-2-oxide (5i). Route (a): Colorless crystals (2.53 g, 66\%); mp 166-169 ${ }^{\circ} \mathrm{C}$. IR (KBr): $\tilde{v} 1224(\mathrm{P}=\mathrm{O}), 1113(\mathrm{O}-$ C), $923(\mathrm{P}-\mathrm{O}) \mathrm{cm}^{-1} .{ }^{1} \mathrm{H}$ NMR (300 MHz, DMSO- $\left.d_{6}\right): \delta 6.34-8.21(\mathrm{~m}, 18 \mathrm{H}), 4.73-5.01(\mathrm{~m}, 4 \mathrm{H})$. ${ }^{13} \mathrm{C}$ NMR $\left(100 \mathrm{MHz}, \mathrm{DMSO}-d_{6}\right): \delta 46.1\left(\mathrm{~d}, J_{\mathrm{PC}}=127.33 \mathrm{~Hz}, \mathrm{C}-4\right), 129.6(\mathrm{C}-4 \mathrm{a}), 128.5(\mathrm{C}-5)$, 124.1 (C-6), 127.7 (C-7), 123.44 (C-8), 152.1 (C-8a), 132.5 (C-1', C-4'), 117.5 (C-2', C-3', C-5', $\left.\mathrm{C}^{\prime} 6^{\prime}\right), 155.4\left(1-\mathrm{C}_{\mathrm{Ar}}\right), 116.3\left(2-\mathrm{C}_{\mathrm{Ar}}\right), 138.3\left(3-\mathrm{C}_{\mathrm{Ar}}\right), 137.1\left(4-\mathrm{C}_{\mathrm{Ar}}\right), 128.5\left(5-\mathrm{C}_{\mathrm{Ar}}\right), 132.1\left(6-\mathrm{C}_{\mathrm{Ar}}\right)$. ${ }^{31} \mathrm{P}$ NMR (161.98 MHz, DMSO-d $\left.d_{6}\right): \delta 1.59$. Anal. calcd. for $\mathrm{C}_{32} \mathrm{H}_{22} \mathrm{Cl}_{4} \mathrm{~N}_{2} \mathrm{O}_{6} \mathrm{P}_{2}: \mathrm{C}, 52.34 ; \mathrm{H}, 3.02$; N, 3.82. Found: C, 52.39; H, 3.11; N, 3.93.

\section{3-[4-[2-(2,6-Dimethylphenoxy)-2-oxo-3,4-dihydro-2 $\lambda^{5}$-benzo[e][1,3,2]oxazaphosphinin-3-} yl]phenyl]-2-(2,6-dimethylphenoxy)-3,4-dihydrobenzo[e][1,3,2] oxazaphosphine-2-oxide (5j). Brown crystals (2.16 g, 64\%); mp 140-142 ${ }^{\circ} \mathrm{C}$. IR (KBr): $\tilde{v} 1222$ (P=O), 1127 (O-C), 917 (P-O) $\mathrm{cm}^{-1} .{ }^{1} \mathrm{H}$ NMR $\left(300 \mathrm{MHz}\right.$, DMSO- $\left.d_{6}\right): \delta 6.41-7.71(\mathrm{~m}, 30 \mathrm{H}), 4.41-4.61(\mathrm{~m}, 4 \mathrm{H}), 1.93-1.99(\mathrm{~m}$, 2,6- $\left.\mathrm{H}_{\mathrm{Ar}}, 12 \mathrm{H}\right) .{ }^{13} \mathrm{C}$ NMR $\left(100 \mathrm{MHz}, \mathrm{DMSO}-d_{6}\right): \delta 45.6\left(\mathrm{~d}, J_{\mathrm{PC}}=128.41 \mathrm{~Hz}, \mathrm{C}-4\right), 129.4(\mathrm{C}-4 \mathrm{a})$, 128.6 (C-5), 124.1 (C-6), 128.0 (C-7), 123.1 (C-8), 152.8 (C-8a), 132.3 (C-1', C-4'), 116.8 (C-2', C-3', C-5', C-6'), $154.3\left(1-\mathrm{C}_{\mathrm{Ar}}\right), 124.3\left(2-\mathrm{C}_{\mathrm{Ar}}\right), 126.4\left(3-\mathrm{C}_{\mathrm{Ar}}\right), 132.4\left(4-\mathrm{C}_{\mathrm{Ar}}\right), 126.4\left(5-\mathrm{C}_{\mathrm{Ar}}\right), 138.7$ $\left(6-\mathrm{C}_{\mathrm{Ar}}\right), 16.3\left(4 \mathrm{C}, 4 \mathrm{CH}_{3}\right) .{ }^{31} \mathrm{P}$ NMR $\left(161.98 \mathrm{MHz}, \mathrm{DMSO}-d_{6}\right): \delta$ 4.62. Anal. calcd. for $\mathrm{C}_{36} \mathrm{H}_{34} \mathrm{~N}_{2} \mathrm{O}_{6} \mathrm{P}_{2}$ : C, 66.25; H, 5.25; N, 4.29. Found: C, 66.39; H, 5.33; N, 4.38.

\section{Acknowledgements}

The authors express their thanks to Dr. C. Naga Raju, Associate Professor, Department of Chemistry, Sri Venkateswara University, Tirupati, for his academic interaction. One of the authors, Dr. CSR thanks DAE (BRNS), Mumbai, India, for providing financial assistance.

\section{References}

1. Prasad, G.; Hari Babu, B.; Kishore Kumar Reddy, K.; Haranath, P. R.; Suresh Reddy, C. Arkivoc 2006, (xiii), 165.

2. Schmidt, B. F.; Tang, W. C.; Eisenbrand, G. Synthesis 1962, 701.

3. Takamizawa, A.; Hamajima, Y.; Mineshita, T.; Hano, Y. S. Jap. Pat. 7016215 (1970); Chem. Abstr. 1970, 73, 66634.

4. Yun, E. S.; Wang, J.S.; Chen, C.T. Heterocycles 1978, 9, 1277.

5. Sosnovsky G.; Paul, B. D. Z. Naturforsch. 1983, 38B, 1146.

6. Reddy, P. V.; Kiran, Y. B.; Reddy C. S.; Reddy, C. D. Chem. Pharm. Bull. 2004, 52, 307.

7. Hill, D. L. A Review of Cyclophosphamide; Thomas: Springfield, IL, 1975.

8. Rubtsova, I. K.; Zhilma, R. D. Zh. Prikl. Khim. 1959, 32, 2604; Chem. Abstr. 1960, 54, 8683. 
9. Bellamy, L. J.; Beecher, L. J. J. Chem. Soc. 1953, 728.

10. Kiran, Y. B.; Govardhan Reddy, P. V.; Devendranath Reddy, C.; Gunasekar, D.; Eswar Reddy, N. P. J. Agric. Food Chem. 2007, 55, 6933.

11. Siva Kumar, B.; Uma Ravi Sankar, A.; Suresh Reddy, C.; Nayak, S. K.; Naga Raju, C. Arkivoc 2007, (xiii), 155.

12. Nyquist, R. A. Appl. Spectrosc. 1957, 11, 161.

13. Thomas L. C.; Chittenden, R. A. Spectrochim. Acta 1964, 20, 489.

14. Kiran, Y. B.; Kasturaiah, M.; Raju, C. N.; Gunasekar, D.; Madhu Babu S. V. S.; Reddy, C. D. Indian J. Chem. 2005, 44B, 2171.

15. Rao, L. N.; Reddy C. D.; Reddy, B. S. Indian J. Chem. 2001, 40B, 817.

16. Cruickshank, R. Medicinal Microbiology, A Guide to Diagnosis and Control of Infection; 11 th ed., E \& S Livingstone: Edinburgh and London, U.K., 1986, p 888.

17. Beuer, A. W.; Kirby, M. M.; Sherries, J. C.; Track, A. Am. J. Clin. Pathol. 1964, 45, 493.

18. Benson, H. J. Microbiological Applications, $5^{\text {th }}$ ed.; W. C. Brown: Dubuque, IA, 1990, p 134. 